\title{
Biomass Characterization Science
}

Arthur J. Ragauskas*

School of Chemistry and Biochemistry, Georgia Institute of Technology

\author{
*Corresponding author: Arthur J. Ragauskas, \\ School of Chemistry and Biochemistry, Institute of Paper Science and Technology, \\ Georgia Institute of Technology, \\ 500 10th St., NW, Atlanta, GA, 30332-0620 \\ E-mail: Art.Ragauskas@ipst.gatech.edu, \\ Received October 26, 2013; Accepted October 28, 2013, Published October 30, 2013.
}

The demand for sustainable technologies has continued to grow as we all become aware of how inter-connected a planet we have become. From climate change to the Pacific trash vortex there are clear long-term challenges to many of our commercial practices. The use of renewable resources is now well acknowledged to be part of this solution. The bio-refining of biomass to biofuels, bio-power, bio-based materials and chemicals has placed new demands on biomass characterization techniques for native and trans-genetic plants and the corresponding products. For example, high throughput determination of biomass constituents is now routinely accomplished by pyrolysis molecular beam mass spectroscopy and is the preferred analysis procedure over older wet-chemistry methods. The structure of lignin and hemicelluloses was historically accomplished using laborious isolation procedures followed often by NMR methods. Today, these methods are being replaced by whole cell NMR of biomass methodology which involves the dissolution of biomass in DMSO or other high solubilization solvents followed by 1D and 2D NMR analysis to characterize the structure of hemicellulose and lignin without the need to isolate the individual components. The analysis of these multi-component spectra has been facilitated by the development of extensive chemical shift correlations that now readily permit structural assignments of most routine subcomponents present in lignin and hemicellulose. Indeed, recent publications have indicated that this process can be automated which facilitates this analysis by non-experts.

The determination of cellulose crystallinity and its individual components including cellulose I $\alpha$, I $\beta$, II, paracrystalline, amorphous, and inaccessible/accessible fibril surfaces can be determined by solid-state NMR. These results provide valuable insight into the ultrastructure of cellulose which has been reported to influence a multitude of cellulose properties from its deconstruction by cellulase to the physical properties of nanocellulose. Along with cellulose crystallinity, research interest has focused on the porosity of biomass and this can be explored by several NMR techniques including NMR cryoporometry, diffusometry, $1 \mathrm{H}$ spin-lattice and $1 \mathrm{H}$ spin-spin relaxometry techniques.

The tagging of functional groups in biomass with hetero-atom groups followed by NMR facilitates the analysis of a host of important functional groups in biomass. For example, phosphitylation of hydroxyl groups followed by 31P NMR allows for the quantitative analysis of hydroxyl groups, phenoxy and carboxylic groups. The concentration of carbonyl groups in lignin can be monitored by derivatization with 4-(trifluromethyl)phenylhydrazine followed by 19F NMR. The use of $2 \mathrm{H} \mathrm{NMR}$ has been used to monitor the chemistry of catalytic hydrodexoygenation of pyrolysis oils. Likewise, the incorporation of deuterium into plants grown in enriched D2O solutions can be readily monitored by whole cell NMR techniques.

The analysis of biomass requires not only structural information but also spatial distribution information. One of the promising techniques to address this challenge is ToF-SIMS which is uniquely well suited to identify the location of lignin and its three basic units guaiacyl, syringyl and p-hydroxyl phenolic. Likewise, MALDI-IMS has been applied to the analysis of the spatial distribution of cellulose on cross-sections of holocellulose poplar.

Although no one technique addresses all the characterization needs of biomass the advances in analytical chemistry coupled with specific tailorization to the chemistry of the plant cell wall polymers has made substantial advances over the past decade. Given these advances and the ability to genetically alter the structure of cellulose, hemicellulose and/or lignin in a given bio-resource, one could envisage the tailoring of plant cell wall chemistry to specific applications and being able to follow chemical transformations from the plant cell wall to final product. Although the need to demonstrate carbon neutrality and bio-degradability of bio-based materials and chemicals is still required the very nature of the starting material lends itself to sustainable practices. Certainly, current advances in biomass characterization will help accelerate these trends. 


\section{References:}

1. Sykes, R.; Yung, M.; Novaes, E.; Kirst, M.; Peter, G.; Davis, M. Biofuels Methods in Molecular Biology, 581 (2009), 169-183.

2. Samuel, R.; Foston, M.; Jaing, N.; Cao, S.; Allison, L.; Studer, M.; Wyman, C.; Ragauskas, A.J. Fuel 90 (2011), 2836-2842.

3. Foston, M.; Ragauskas, A. J. Biomass and Bioenergy (2010), 34(12), 1885-1895.

4. Meng, X.; Kosa, M.; Ragauskas, A.J. Bioresource Technology (2013), 144, 467-476.

5. Pu Y.; Cao S.; Ragauskas A.J., Energy \& Environmental Science (2011), 4(9), 3154-316.

6. Ben, H.; Ferguson, G.A.; Mu, W.; Pu, Y.; Huang, F.; Jarvis, M.; Biddy, M; Deng, Y.; Ragauskas, A.J., Phys. Chem. Chem. Phys. (2013), 15, 19138 - 19142.

7. Bali, G.; Foston, M.B.; O'Neill, H.M.; Evans, B. R.; He, J.; Ragauskas, A.J., Carbohydrate Research (2013), 374, 82-88.
8. Jung, S.; Foston, M.; Kalluri, U.C.; Tuskan, G.A.; Ragauskas, A.J., Angewandte Chemie, International Edition (2012), 51(48), 12005-12008.

9. Seokwon J.; Yanfeng C.; Cameron, S.M.; Ragauskas, A.J. Rapid Communications in Mass Spectrometry (2010), 24(10), 3230-3236.

Professor Ragauskas held the first Fulbright Chair in Alternative Energy at Chalmers University of Technology/Sweden and is a Fellow of American Association for the Advancement of Science Fellow, the International Academy of Wood Science and TAPPI. His research program at Georgia Institute of Technology is seeking to understand and exploit innovative sustainable bio-resources via sustainable bio-refining technologies.

Dr. Ragauskas received his PhD in Chemistry in 1985 at the University of Western Ontario, and after post-doctoral research studies at the University of Alberta and Colorado State University, he assumed a position at the National Research Council of Canada as a n Associate Research Scientist. Then in 1989 he moved to the Institute of Paper Science and Technology in Atlanta GA and in 2003 he joined Georgia Institute of Technology in the School of Chemistry and Biochemistry. 